Cite this: Soft Matter, 2014, 10, 2693

Received 2nd January 2014

Accepted 20th February 2014

DOI: $10.1039 / \mathrm{c} 4 \mathrm{sm} 00009$ a

www.rsc.org/softmatter

\section{Charge and sequence effects on the self-assembly and subsequent hydrogelation of Fmoc- depsipeptides $\uparrow$}

\begin{abstract}
Mary M. Nguyen, Kevin M. Eckes and Laura J. Suggs*
Herein we report on the self-assembly of a family of Fmoc-depsipeptides into nanofibers and hydrogels. We show that fiber formation occurs in depsipeptide structures in which the fluorenyl group is closely associated and that side-chain charge and sequence affect the extent of self-assembly and subsequent gelation. Using fluorescence emission spectroscopy and circular dichroism, we show that self-assembly can be monitored and is observed in these slow-gelling systems prior to hydrogel formation. We also demonstrate that the ionic strength of salt-containing solutions affects the time at which self-assembly results in gelation of the bulk solution. From transmission electron microscopy, we report that morphological changes progress over time and are observed as micelles transitioning to fibers prior to the onset of gelation. Gelled depsipeptides degraded at a slower rate than non-gelled samples in the presence of salt, while hydrolysis in water of both gels and solution samples was minimal even after 14 days. Our work shows that while incorporating ester functionality within a peptide backbone reduces the number of hydrogen bonding sites available for forming and stabilizing supramolecular assemblies, the substitution does not prohibit self-assembly and subsequent gelation.
\end{abstract}

\section{Introduction}

Peptide-based structures have advantages as biomaterials because of the potential for controlled biologic function. ${ }^{1-3}$ Based on their ability to form higher-ordered structures, they are also able to self-assemble into self-supporting gels, which have application as potential injectable carriers in drug delivery $^{4}$ and tissue engineering. ${ }^{5,6}$ The designer self-complementary peptides, $\mathrm{EAK},{ }^{7,8} \mathrm{RAD}^{7,8}$ and $\mathrm{KFE},{ }^{9,10}$ have been well cited in the literature, and their chemical structures alternate hydrophobic and hydrophilic domains which influence the selfassembly into nanofibers, nanotubes, or hydrogels. ${ }^{5,6,11-14}$ Selfassembly is influenced by the length, sequence, and composition of the chemical backbone within specific environmental conditions, and predicting these processes is not straightforward. For example, KFE8 and KFE16 exhibit similar gelation conditions, while KFE12, with an intermediate length, requires a lower concentration of salt to form a gel. Consistent with the EAK and RAD peptide assembly, each molecule must pack together as a beta-strand with other oligopeptides to form a

Department of Biomedical Engineering, The University of Texas at Austin, 107 W Dean Keeton Street, Austin, TX, 78712,USA.E-mail:m.nguyen@utexas.edu; suggs@utexas. edu; Fax: +1512471 0616; Tel: +1 5122328593

$\dagger$ Electronic supplementary information (ESI) available: Figures of the following: fluorescence emission spectra of Fmoc-amino acids and Fmoc-depsidipeptides, results of a degradation study of Fmoc-D-Lac-K-A-8 in PBS and mass spectra of degradation products, and fluorescence spectra of Fmoc-D-Lac-K-A-16. See DOI: 10.1039/c4sm00009a beta-sheet, suggesting that KFE8 is not long enough to maintain this structure at low salt concentrations. On the other hand, KFE16 peptides have more bonds and therefore more degrees of freedom. Thus, bond rigidity is increased to stabilize the betastrand and therefore entropy is decreased, causing a decrease in the propensity to self-assemble at lower salt concentrations.

Self-assembly has also been studied on short peptide structures with conjugated aromatic groups at the N-terminal, such as the Fmoc moiety. ${ }^{15}$ Fmoc-peptides are relatively small molecules, with a molecular weight of less than $1000 \mathrm{Da}$ and usually self-assemble in water to form long, fibrous structures. Fmocpeptides are very robust in their ability to self-assemble and subsequently form gels. While the assembly of Fmoc-peptides is not well-understood, it has been proposed that the hydrophobicity and planar character of the Fmoc group, the ability of the peptide chain to hydrogen bond, and interactions among adjacent peptide residues may all contribute to the formation of nanostructured fibers and ultimately to hydrogels. ${ }^{16-21}$ For example, a library of seven Fmoc-dipeptides were evaluated for successful gelation, and results indicate that gelation was highly dependent on the interaction of the fluorenyl groups, as circular dichroism spectrum of all gels gave rise to peaks indicative of $\pi-\pi^{*}$ transitions in the Fmoc structure. In samples that did not form gels, no contribution from the aromatic region was observed with spectroscopic methods. ${ }^{22}$ In a separate study, Fmoc-Phe-Gly forms a gel under 2 conditions while a simple change in the sequence to Fmoc-Gly-Phe does not. ${ }^{16}$ Our group has previously demonstrated both experimentally and 
computationally that while the stacking of the Fmoc group of Fmoc-Ala-Ala was critical in fiber formation and subsequent gelation, the formation of $\beta$-sheet-like hydrogen bonds between molecules was not prevalent. ${ }^{23}$

The structure and function of short self-complementary peptides can be altered upon modification of the native peptide backbone. ${ }^{24}$ Altering the peptide backbone with esters to form depsipeptides, can confer properties such as faster degradation while potentially preserving biologic properties, ${ }^{25-27}$ selfassembly, ${ }^{28,29}$ and hydrogel formation. ${ }^{25}$ Incorporating esters into the peptide backbone reduces the potential for hydrogen bonding interactions, however, linear depsipeptides based on amylin sequences have been reported to self-assemble into helical ribbons and peptide nanotubes. ${ }^{29}$ Amylin(20-29) derivatives were modified with esters at positions 24,26 , and 28; self-assembly of the depsipeptides was unexpected, as a single ester substitution prevented gelation. The depsipeptide did not exhibit $\beta$-sheet conformation as is traditionally observed for amylin fibers, suggesting that folding other than $\beta$-sheet character is responsible for the self-assembly.

In an effort to capitalize on both the robust assembly and gelation of Fmoc-peptides as well as the hydrolytic sensitivity of depsipeptides, we are interested in developing Fmoc-depsipeptides as a class of biomaterials. Furthermore, we propose to use these materials to investigate the role of inter-chain hydrogen bonding, side-chain charge, and sequence specificity on selfassembly and gelation. The goal of this work is to demonstrate the self-assembly of Fmoc-depsipeptides into nanofibers and hydrogels. We show that the depsipeptide side chain charge and sequence affect the extent to which nanostructured supramolecular fibers form. We also investigated how the ionic strength of salt-containing solutions affects the time at which selfassembly results in gelation of the bulk solution.

\section{Materials and methods}

\section{Depsipeptide design}

The depsipeptides developed by our group were inspired by both short, self-complementary peptides ${ }^{5,12,14,30-35}$ and Fmocdipeptides. ${ }^{\text {20,21,36,37 }}$ The depsipeptide library was synthesized with standard Fmoc-solid phase methods with depsi-dipeptides as the coupling building block (Fig. 1). ${ }^{38}$ The depsipeptides have an alternating sequence of hydrophilic and hydrophobic residues; they are composed of di-depsipeptide units of lysine (K) or aspartic acid (D) and lactic acid (Lac). The charges are aligned in a block-like manner for structures containing $\mathrm{K}$ and $D$ residues, and an alanine (A) or phenylalanine (F) residue was positioned at the C-terminal to reduce undesired side-products. $^{38}$ The depsipeptides were named according to the $\mathrm{N}$ terminal amino acid and the linear order of various residues followed by the total number of residues. The design strategy of alternating hydrophobic Lac residues with the charged residues $\mathrm{K}$ and $\mathrm{D}$ was adapted from the sequence of the EAK16 peptide, which has a hydrophobic alanine (A) residue that alternates with either the hydrophilic glutamic acid (E) or lysine (K) amino acids, AEAEAKAKAEAEAKAK. We were also interested in investigating how the Fmoc group influences the self-assembly of our depsipeptide library. Many Fmoc-dipeptides have been found to
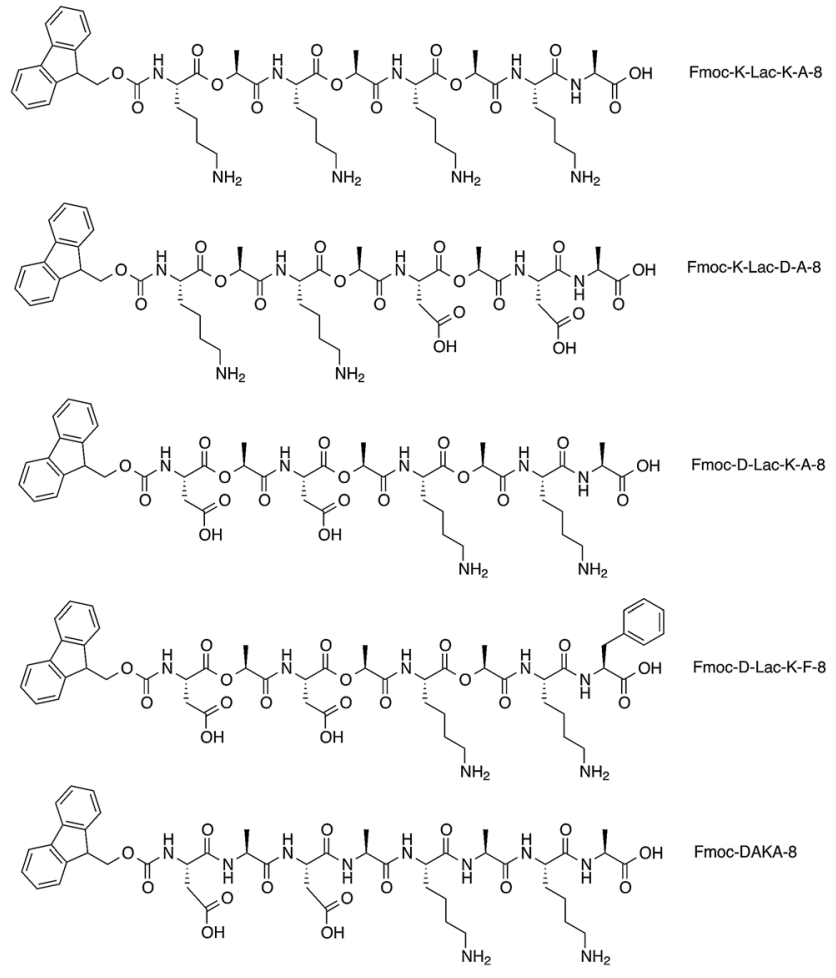

Fig. 1 Depsipeptide library. The depsipeptides were named according to the $\mathrm{N}$-terminal amino acid and the order of various residues, where $\mathrm{K}$ is lysine, Lac is lactic acid, $\mathrm{D}$ is aspartic acid and $\mathrm{A}$ or $\mathrm{F}$ is the residue at the $\mathrm{C}$-terminus, alanine or phenylalanine respectively. The shorthand name is followed by the total number of residues. Fmoc-DAKA- 8 is the peptide equivalent of Fmoc-D-Lac-K-A-8.

self-assemble into ordered structures, ${ }^{37}$ and spectral changes due to the association and orientation of fluorenyl groups within self-assembled systems have been well-cited using fluorescence spectroscopy ${ }^{21,39}$ and circular dichroism. ${ }^{22,28}$

\section{Depsipeptide synthesis}

Abbreviations: Boc, tert-butyloxycarbonyl; $\mathrm{O}$ tBu, tert-butyl; $\mathrm{D}_{2} \mathrm{O}$, deuterium oxide; DCM, dichloromethane; DMAP, 4-dimethylaminopyridine; DMF, dimethylformamide; DIC, $N, N^{\prime}$-diisopropylcarbodiimide; Fmoc, 9-fluorenylmethyloxycarbonyl; TFA, trifluoroacetic acid; TIPS, triisopropylsilane. TFA, DCM, DMF, and diethyl ether were from Fisher Scientific. DIPEA was purchased from Acros Organics. $\mathrm{D}_{2} \mathrm{O}$ was from Cambridge Isotope Laboratories, Inc. Fmoc-amino acids and DMAP were purchased from EMD Biosciences. Fmoc-Phe-Wang resin, Fmoc-Ala-Wang resin, and DIC were from Anaspec. Piperidine was from Alfa Aesar. TIPS was purchased from Acros.

Fmoc-K-Lac-8, Fmoc-K-Lac-D-A-8, and Fmoc-D-Lac-K-A-8 were prepared as reported in earlier work. ${ }^{38}$ Fmoc-D-Lac-K-F-8 was prepared using the same methods with the exception of first coupling to an Fmoc-Phe-Wang resin (30\%). Fmoc-DAKA-8 was synthesized with manual solid phase peptide synthesis methods (37\%). Fmoc-Ala-Wang resin was coupled to Fmoc-Lys(Boc)-OH in DMF and DCM with DIC and DMAP. After 2 hours, the resin was washed with DMF and DCM. Upon removing the Fmoc group in $20 \%$ piperidine in DMF, Fmoc-Ala-OH in DMF and DCM was 
coupled to the resin with DIC and DMAP. After 2 hours, the resin was washed with DMF and DCM. The process of removing the Fmoc-group, coupling with Fmoc(Boc)-OH, Fmoc-Ala-OH, or Fmoc-Asp $(\mathrm{O} t \mathrm{Bu})-\mathrm{OH}$, and washing with DMF and DCM was repeated until the desired sequence was obtained. The peptide was cleaved with TFA/water/TIPS (95:2:3) for 3 hours, precipitated in diethyl ether, and purified with HPLC. The depsipeptides were characterized using liquid chromatography-mass spectrometry (LC-MS) employing electrospray ionization (ESI).

\section{Sample preparation}

Samples were dissolved in either pure distilled and deionized water, phosphate buffered saline (PBS), or PBS with an additional $10 \mathrm{mM}$ sodium chloride (PBS $+\mathrm{NaCl})$. PBS without calcium and magnesium was used and had a concentration of $\mathrm{NaCl}$ of $137 \mathrm{mM}$ before additional salt was added. Unless otherwise specified, samples were prepared at a concentration of $10 \mathrm{mg} \mathrm{ml}^{-1}$. The $\mathrm{pH}$ of the PBS and PBS + $\mathrm{NaCl}$ bulk solutions were adjusted with $0.1 \mathrm{~N} \mathrm{HCl}$ or $0.1 \mathrm{~N} \mathrm{NaOH}$. The samples were kept at room temperature and visually observed for hydrogel formation every 18-24 hours. Gelation was defined as the inability of the solution to flow under vial inversion.

\section{Stability study}

The hydrolysis products of Fmoc-D-Lac-K-A-8 were observed over 14 days at room temperature using HPLC-mass spectrometry. The Fmoc-depsipeptide formed a gel in water, PBS, and PBS $+\mathrm{NaCl}$ at a concentration of $10 \mathrm{mg} \mathrm{ml}^{-1}$ over 3 weeks, 6 days, and 2 days respectively. Samples were analyzed postgelation. After 4, 7, 10, and 14 days, $100 \mu \mathrm{l}$ was collected and diluted in $900 \mu \mathrm{l}$ of respective solution. Non-gelled samples were prepared under the critical gel concentration of $1 \mathrm{mg} \mathrm{ml}^{-1}$ and read after $4,7,10$, and 14 days.

\section{Fluorescence experiments}

Fluorescence emission spectra were collected on a Molecular Devices plate reader provided by the DNA Sequencing Facility at the Institute of Cellular and Molecular Biology. Samples were read in 96-well black plates with an excitation of $265 \mathrm{~nm}$ from 300-500 $\mathrm{nm}$ and the respective solution background was subtracted from sample spectrum prior to data analysis.

\section{Transition emission microscopy (TEM)}

Images were obtained using a FEI Tecnai Transmission electron microscope operating at an acceleration voltage of $80 \mathrm{kV}$. Samples $(5 \mu \mathrm{l})$ were placed onto glow-discharged or untreated carbon coated copper grids for 10 seconds, removing the excess with filter paper. Water $(5 \mu \mathrm{l})$ was placed on grids containing samples prepared in PBS or PBS $+\mathrm{NaCl}$. This process was repeated twice, and the samples were then negatively stained with uranyl acetate $(5 \mu \mathrm{l})$.

\section{Fourier transform infrared spectroscopy (FTIR)}

FTIR experiments were performed with dry or wet samples on a Bruker Vertex 70 FTIR spectrometer provided by the Webb group in the Department of Chemistry at the University of Texas at Austin. The spectrometer was equipped with an A518/Q horizontal reflection cell (Bruker) for illuminating the sample at a grazing angle of $80^{\circ}$ with respect to the surface. The reflection cell was purged with $\mathrm{N}_{2}$ for 1 hour prior to starting experiments and handled by methods reported in the literature. ${ }^{40}$ Spectra were recorded using a mercury cadmium telluride (MCT) detector, with 100-400 scans between 400 and $4000 \mathrm{~cm}^{-1}$ at a resolution of $4 \mathrm{~cm}^{-1}$. Samples were also analyzed using an ATR cell equipped with a germanium crystal. The sample substrates consisted of silicon wafers coated with chromium and gold, which were generously prepared and provided by the Webb group. The surfaces were cleaned with either hydrogen flame annealing or with a piranha wash. The latter method was followed by rinses in concentrated $\mathrm{HCl}$, high purity water, and ethanol, then dried with $\mathrm{N}_{2}$. Fmoc-D-Lac-K-A-8 gelled in water, $\mathrm{PBS}$, and $\mathrm{PBS}+\mathrm{NaCl}$ at a concentration of $10 \mathrm{mg} \mathrm{ml}^{-1}$ over 3 weeks, 6 days and 2 days, respectively, prior analysis. The samples were dried overnight on clean wafers and analyzed by placing the sample side of the wafer face down on the reflection (or ATR) cell crystal. Non-gelling solutions of Fmoc-D-Lac-K-A was dissolved in water, PBS, or PBS $+\mathrm{NaCl}$, immediately placed on the wafer, and dried overnight. Samples prepared in PBS + $\mathrm{NaCl}$ caused extensive crystallization of salts upon drying; these samples were thus analyzed wet. To analyze non-self-assembled samples, a freshly prepared sample $\left(20 \mu \mathrm{l}, 10 \mathrm{mg} \mathrm{ml}^{-1}\right)$, mixed less than 2 minutes prior to measurement, was placed on the cell and sandwiched with a clean wafer. Fmoc-D-Lac-K-A-8 gelled in $\mathrm{PBS}+\mathrm{NaCl}$ at a concentration of $10 \mathrm{mg} \mathrm{ml}^{-1}$ over 2 days prior analysis. Gelled Fmoc-DAKA-8 was prepared at a concentration of $10 \mathrm{mg} \mathrm{ml}^{-1}$ in water and aged at least 2 days. Turbidity was observed instantly upon dissolving Fmoc-DAKA-8 in water, thus it was not possible to investigate a pre-assembled state by taking scans soon after mixing. Adding $1.0 \mathrm{~N} \mathrm{NaOH}$ to adjust the pH of Fmoc-DAKA-8 in water yielded a non-gelled state, which was used for analysis in solution. Scans of the respective solution served as the background spectrum, which was subtracted from sample spectra.

\section{Circular dichroism (CD)}

CD was collected on a Jasco J-815 CD Spectrometer. Fmoc-DLac-K-A-8 was dissolved in PBS + NaCl, PBS, and water at $10 \mathrm{mg}$ $\mathrm{ml}^{-1}$ and aged for 4 days. Fmoc-DAKA-8 was dissolved in PBS + $\mathrm{NaCl}$, PBS, and water at $10 \mathrm{mg} \mathrm{ml}^{-1}$ initially and aged for 24 hours. The concentrations in PBS $+\mathrm{NaCl}$ and PBS were adjusted to bring the mixture to dissolution. The sample (50-100 $\mu \mathrm{l})$ was pipetted onto a cylindrical cuvette $(0.1 \mathrm{~mm})$ and carefully sandwiched as to avoid air bubbles. CD spectra were measured over the range $180-350 \mathrm{~nm}$ in with a scanning speed of $200 \mathrm{~nm}$ $\min ^{-1}$, data pitch of $1 \mathrm{~nm}$, and 3 accumulations. The optical chamber was continually flushed with nitrogen.

\section{Rheometry}

Depsipeptide solutions were prepared and allowed to gel in hollow cylindrical molds with a diameter of $8 \mathrm{~mm}$. The gelled samples were carefully removed and placed on the stage of the 
rheometer. Measurements were collected on an Anton Paar Physica MCR 101 rheometer using an $8 \mathrm{~mm}$ diameter parallel plate geometry at room temperature and a fixed gap of 0.8-1.30 $\mathrm{mm}$. The storage and loss moduli were measured with a frequency sweep test $(0.1-100 \mathrm{~Hz})$ with a fixed strain amplitude $(1.0 \%)$ and were averaged over 4-5 samples.

\section{Results and discussion}

\section{Spectroscopic and morphological analysis of depsipeptide self-assembly}

We investigated excimer formation of the fluorenyl group as an indicator of close association and self-assembly among the depsipeptides Fmoc-K-Lac-K-A-8, Fmoc-K-Lac-D-A-8, and FmocD-Lac-K-A-8. The fluorescence emission spectrum of Fmoc-KLac-K-A-8 in PBS or PBS + NaCl within the $\mathrm{pH}$ range of 6.0-9.0 shows a peak at $310 \mathrm{~nm}$ (Fig. S1A $\dagger$ ), which is comparable to the fluorescence emission spectra collected on non-assembled Fmoc-peptides: Fmoc-Lys(Boc)-OH, Fmoc-Asp(OtBu)-OH, FmocLys(Boc)-Lac-OH, and Fmoc-Asp(OtBu)-Lac-OH (Fig. S1B $\dagger$ ). These results suggest the peak at $310 \mathrm{~nm}$ is the emission maximum of the fluorenyl group of each Fmoc-depsipeptide in its monomeric state, and does not correlate to a state of ordered assembly.

The emission spectra of Fmoc-K-Lac-D-A-8 in ionic solutions 6 days after preparation show a shoulder peak centered around $370 \mathrm{~nm}$ that is absent in water (Fig. 2A). TEM images of this depsipeptide show the formation of unordered aggregates in PBS and PBS $+\mathrm{NaCl}$ after 11 days (Fig. 2B), while a mixture of globular structures approximately $75 \mathrm{~nm}$ in diameter and short fiber-like structures 200-350 $\mathrm{nm}$ long are observed in water. Taken with the emission spectra, this result indicates that in water, Fmoc-K-Lac-D-A-8 may undergo self-assembly to some extent. The shoulder peak of the fluorescence spectra at $370 \mathrm{~nm}$ has been correlated with extensive aromatic stacking of fluorenyl groups, ${ }^{8}$ which has been reported to contribute to the selfassembly of certain Fmoc-dipeptides into micelles. ${ }^{21,39}$ In our system, it is clear some self-assembly has taken place but has not resulted in regular micelle-like structures; it is possible that the increased number of residues or steric hindrance due to large lysine side chains adjacent to the fluorenyl groups prevents formation of true micelles. These results also indicate the association of the fluorenyl groups is influenced by depsipeptide sequence.

Excimer formation between multiple aromatic ring structures, such as the Fmoc group, is readily investigated with fluorescence emission spectroscopy. Prior work with covalentlybound fluorine dimers demonstrated different emission peak energies depending on syn- or anti-arrangement. ${ }^{41}$ Multiple groups have inferred specific orientations for Fmoc groups during the self-assembly process of supramolecular fibrils, such as $\pi$-stacking, anti-parallel, or parallel association of the fluorenyl structures..$^{\mathbf{2 0 , 4 2}}$ Recent work has demonstrated that extensive, close aromatic stacking produces the most dramatic red shift while other, less efficient modes of aromatic overlap produce a smaller red shift over that of the monomer. ${ }^{43,44}$ In comparing the fluorescence emission spectrum of Fmoc-K-LacK-A-8 (Fig. S1A $\dagger$ ) with Fmoc-K-Lac-D-A-8, our results suggest that the presence of aspartic acid in the latter depsipeptide facilitates closer interaction, and possibly excimer formation, between fluorenyl groups.

In contrast to Fmoc-K-Lac-D-A-8, Fmoc-D-Lac-K-A-8 selfassembled to ultimately form a hydrogel. Gelation was defined as the inability of the solution to flow under vial inversion, and the depsipeptide formed a hydrogel in water, PBS, and PBS + $\mathrm{NaCl}$ over 3 weeks, 6 days, and 2 days respectively (Table 1). After 2 days in PBS + NaCl, a red shift from $310 \mathrm{~nm}$ to $320 \mathrm{~nm}$ was observed with fluorescence spectroscopy (Fig. 3A). Similarly, the peak at $320 \mathrm{~nm}$ is observed in the PBS solution after 6 days (Fig. 3B), consistent with the time at which gelation was observed. Fmoc-DAKA-8, the peptide analogue of Fmoc-D-Lac$\mathrm{K}-\mathrm{A}-8$, was also evaluated and demonstrated a similar fluorescence shift in water (Fig. 3C).

The morphology of Fmoc-D-Lac-K-A-8 pre- and post-gelation was observed with TEM after 18 hours and 4 days. The study was designed to observe intermediate self-assembled structures prior to gelation, as gelation occurred after 2 days in $\mathrm{PBS}+\mathrm{NaCl}$, 6 days in PBS, and over 3 weeks in water. After 18 hours, fibers

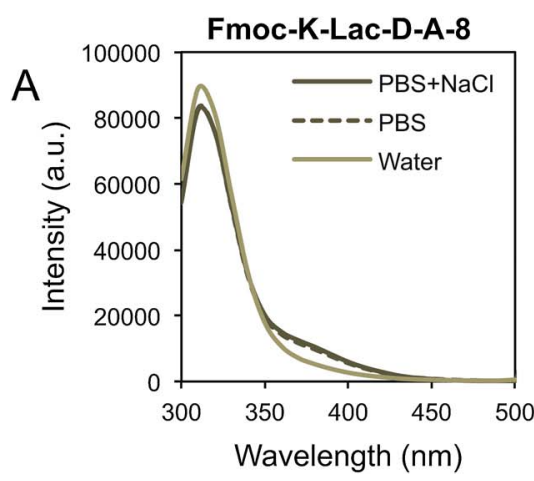

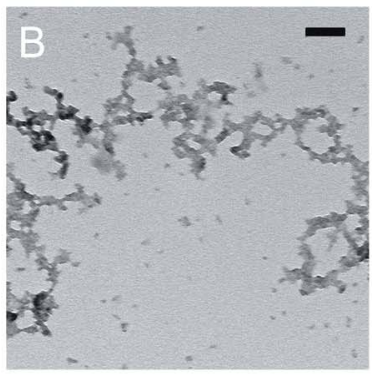

$\mathrm{PBS}+\mathrm{NaCl}$

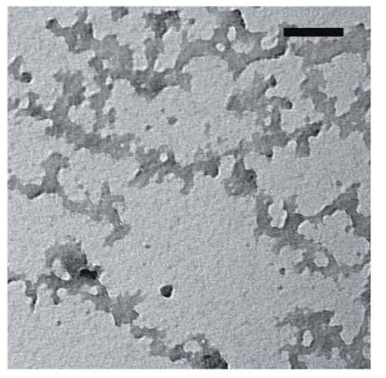

PBS

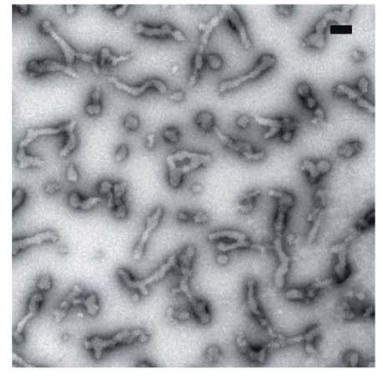

Water

Fig. 2 Fluorescence emission spectra (A) and TEM (B) of Fmoc-K-Lac-D-A-8. When prepared in PBS $+\mathrm{NaCl}$ and PBS at $10 \mathrm{mg} \mathrm{ml}^{-1}$, a broad shoulder around $370 \mathrm{~nm}$ is observed, whereas in water the shoulder peak is not as pronounced, perhaps indicating a small degree of selfassembly. Emission spectra displayed in (A) were collected 6 days after sample preparation. TEM samples were stained with uranyl acetate, and the collected images show globular, unordered networks in ionic solutions. Short, fiber-like morphologies are observed only in aqueous solutions, indicating some self-assembly has taken place. Scale bar $=100 \mathrm{~nm}$. 
Table 1 Summary of gelation results for Fmoc-D-Lac-K-A-8. Gelation is defined as the inability of the material to flow upon inversion and takes the shape of the vial. Fmoc-D-Lac-K-A-8 in PBS $+\mathrm{NaCl}$ is shown on the right

\begin{tabular}{lll}
\hline Solvent & Calculated ionic strength in bulk $(\mathrm{mM})$ & Time hydrogel observed \\
\hline PBS $+\mathrm{NaCl}$ & 175 & 2 days \\
PBS & 165 & 6 days \\
Water & $1.46 \times 10^{-4}$ & $>3$ weeks
\end{tabular}
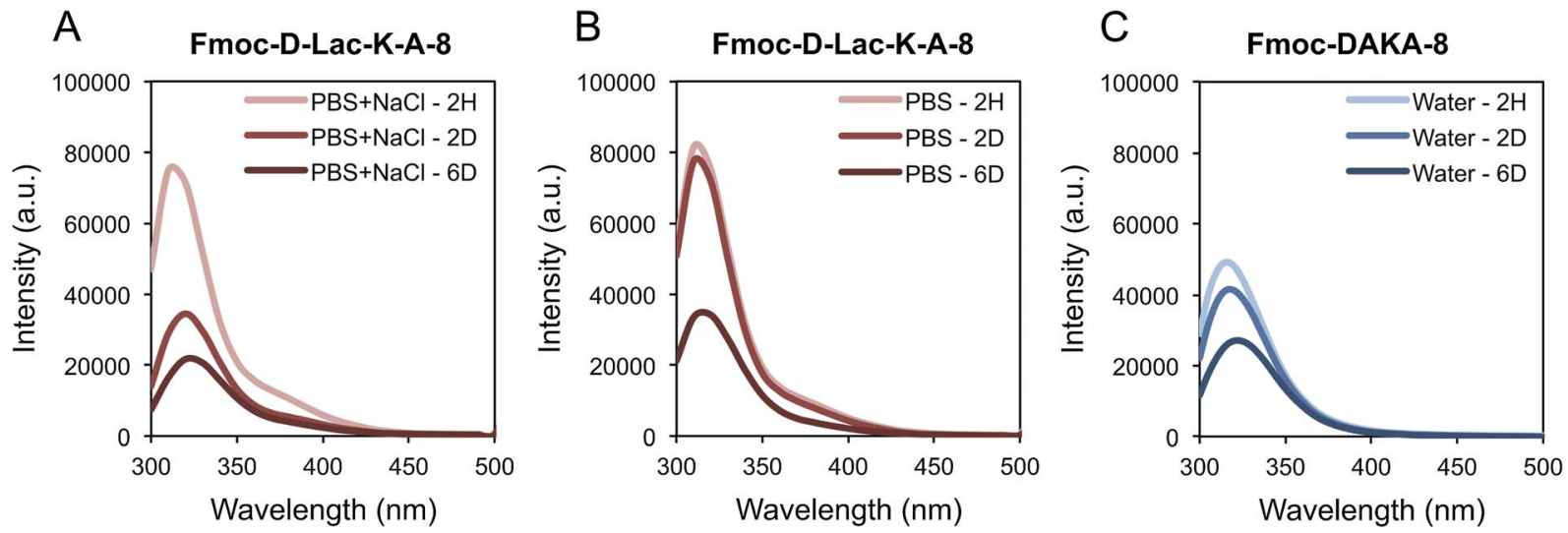

Fig. 3 Fluorescence emission spectra of Fmoc-D-Lac-K-A-8 in PBS + NaCl (A) and PBS (B) were collected after 2 hours, 2 days, and 6 days. In PBS $+\mathrm{NaCl}$, both a red shift from 310 to $320 \mathrm{~nm}$ and decrease in intensity is observed upon the formation of a gel after 2 days. Similarly, the same trend is observed in PBS after 6 days. The peptide equivalent, Fmoc-DAKA- 8 forms a gel in water overnight; emission spectra were collected after 2 hours, 2 days, and 6 days in water (C).

were observed in PBS and PBS + NaCl samples, while spherical aggregates were present in water (Fig. 4A-C). After 4 days, fibers are observed in all three solutions (Fig. 4D-F), similar to fibrous structures seen in Fmoc-DAKA-8 gels at the same time (Fig. S2†).
Compared to the co-assembly of short, oppositely charged Fmoc-peptide hydrogels, ${ }^{19}$ in which the anti-parallel stacking of the fluorenyl groups resulted in the formation of nanofibers and ultimately supramolecular hydrogels, the Fmoc-depsipeptide
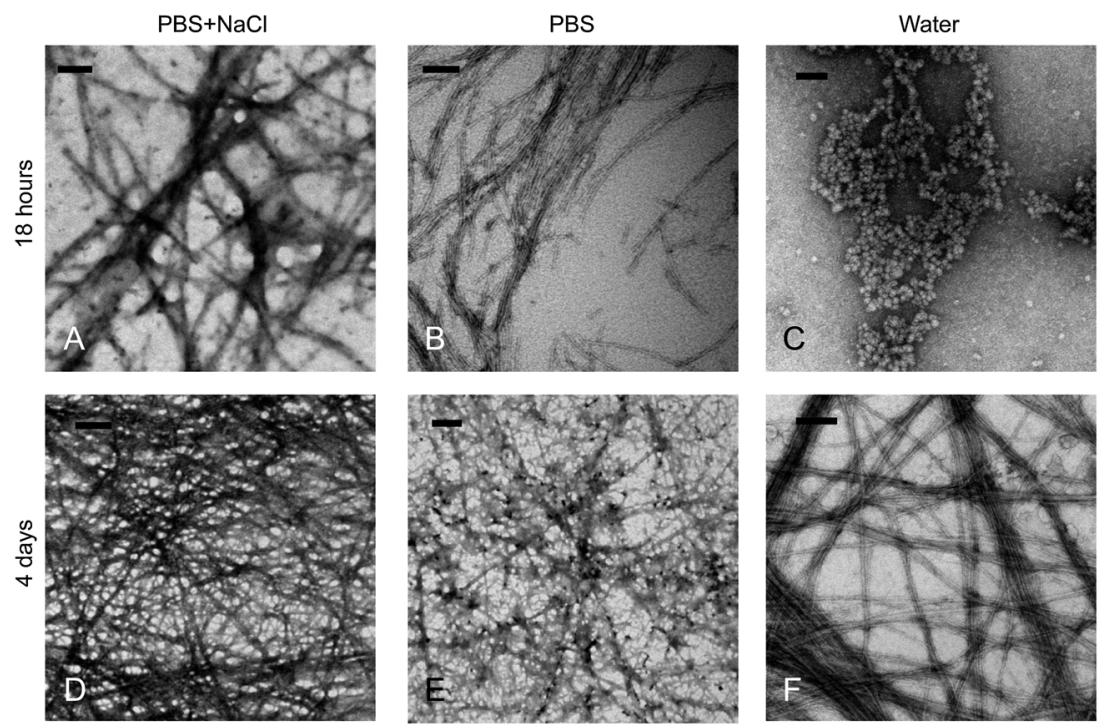

Fig. 4 TEM images of Fmoc-D-Lac-K-A-8. The depsipeptide was prepared at $10 \mathrm{mg} \mathrm{ml}^{-1}$ in PBS + NaCl (A and D), PBS (B and E), and water (C and F). The images were collected after 18 hours ( $A-C$, scale bar $=100 \mathrm{~nm}$ ) and after 4 or 5 days (D-F, scale bar $=200 \mathrm{~nm})$. After 18 hours, relatively short fiber networks are observed in PBS $+\mathrm{NaCl}$ and PBS, while globular aggregates are shown in water. After 4 or 5 days, fibers are observed for all samples. 

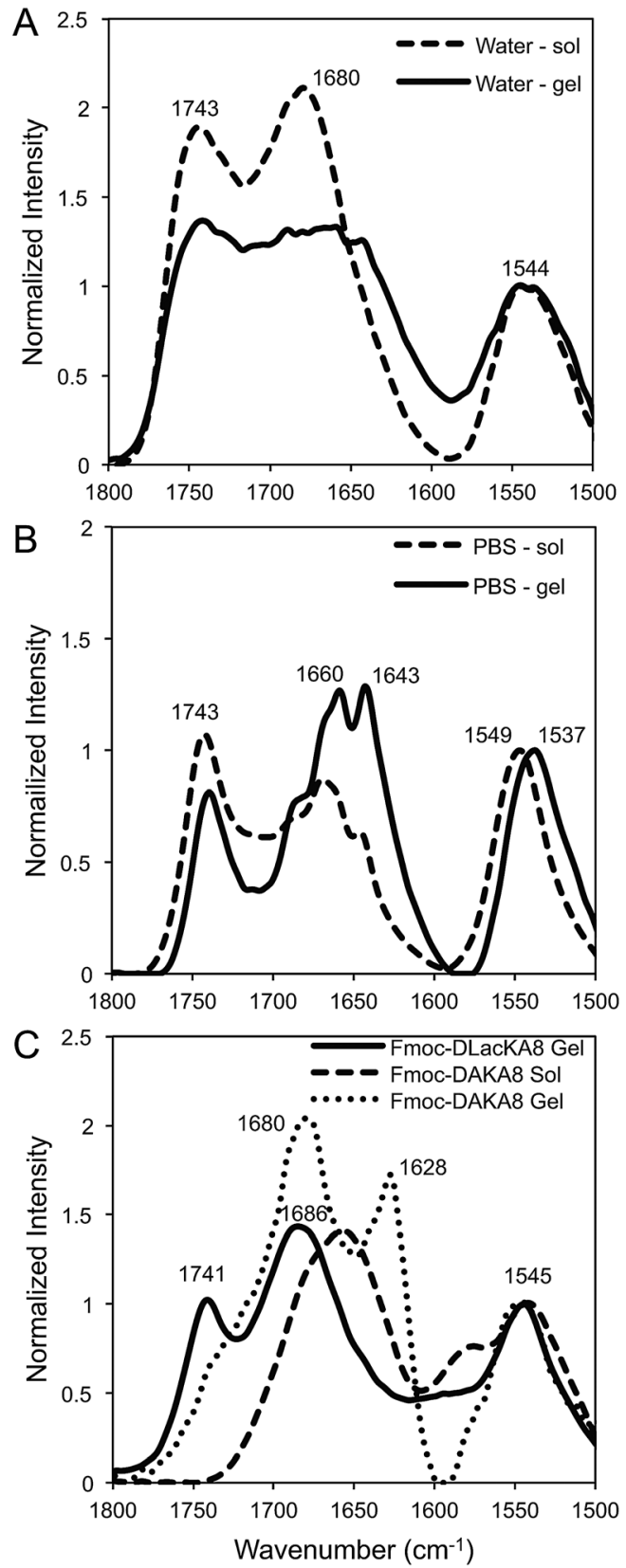

Fig. 5 FTIR spectra of Fmoc-D-Lac-K-A-8. The depsipeptide was prepared in water (A) and PBS (B) in solution and in gelled form. In water, the peaks pre- and post-gelation are relatively similar. In PBS, a splitting pattern in the gelled sample at 1660 and $1643 \mathrm{~cm}^{-1}$ is observed The depsipeptide gel was prepared in PBS $+\mathrm{NaCl}$ and compared to the peptide equivalent, Fmoc-DAKA-8 (C) in solution or as a hydrogel. All spectra were normalized to the amide II absorption peak at $1545 \mathrm{~cm}^{-1}$.

nanofibers were observed under all conditions after 4 days. However, the less highly stacked positioning of the Fmocgroups, as evidenced by fluorescence emission, was only evident in PBS $+\mathrm{NaCl}$ (Fig. 3A), at which point a gel had already formed. Correlating the association of the fluorenyl groups to the selfassembly of fiber networks observed in Fmoc-peptides, ${ }^{21,39}$ our results suggest that the efficiency of aromatic overlap in the Fmoc-excimer is solution-dependent.
Spectral changes occur upon self-assembly of both fibers and hydrogels, although spectral changes over time are more pronounced around the gelation point; therefore, conditions under which self-assembly occurs, and therefore the emission spectra evolve, more quickly over time also cause a more rapid onset of gelation. As a comparison, Fmoc-DAKA-8 was observed to form a viscous, slightly turbid solution within minutes after mixing with water, indicating initial self-assembly. Fmoc-DAKA8 self-assembled to a gel-forming density after 18 hours. The emission spectrum (Fig. 3C) is consistent with those of the gelled samples of the depsipeptide. The spectral shift to $320 \mathrm{~nm}$ is characteristic of less efficient aromatic overlap attributed to anti-parallel alignment of fluorenyl groups for Fmoc-dipeptides. ${ }^{21}$ Within our system, less efficient overlap of the Fmoc groups is observed in gelled samples, and similar emission trends have been reported in Fmoc-peptide families post-gelation. $^{20,21,45}$ In water, self-assembly leads to critical gelation density only after 3 weeks. During the initial stages of assembly, it is possible that the fluorenyl group transitions from an orientation with a very high degree of stacking to a much less ordered arrangement. The process may influence the formation of micelle-like structures, which then aggregate to form fibrils in which Fmoc-groups are associated mainly within the core of the fiber. The transition into fibers causes an increase in intensity as the Fmoc groups are no longer quenched, and over time, the decrease of the fluorescence emission at $320 \mathrm{~nm}$ indicates the self-assembly into a fiber network..$^{21,39}$

The chemical structure of our depsipeptides are designed with alternating esters within a native peptide backbone, thus the effects of hydrogen bonding are reduced. Given that selfassembly is modulated by a number of non-covalent interactions, including hydrogen bonding, Fmoc-D-Lac-K-A-8 was investigated at both early and late stages of self-assembly with IR spectroscopy in water (Fig. 5A) and PBS (Fig. 5B). Results show very little difference between the pre-gelled and postgelled samples in water, which have peak positions at 1743 , 1680 , and $1544 \mathrm{~cm}^{-1}$, referring to the ester carbonyl stretch, amide I and amide II band, respectively. Similar peak patterns are observed between the pre- and post-gelled samples in PBS, with the exception of a splitting pattern at 1660 and $1643 \mathrm{~cm}^{-1}$ in the hydrogel. Fmoc-D-Lac-K-A-8 in PBS + NaCl post-gelation was compared to Fmoc-DAKA-8 in solution and a hydrogel (Fig. 5C), as described in the methods section. The depsipeptide hydrogel shows peak positions around 1741, 1686, and $1545 \mathrm{~cm}^{-1}$, which is consistent with the data observed in water. The IR spectrum of Fmoc-DAKA-8 in a non-gelled form shows sharp peaks at 1657 and $1543 \mathrm{~cm}^{-1}$ with a broad shoulder around $1579 \mathrm{~cm}^{-1}$. In gelled form, the peptide equivalent has sharp peaks at 1680 and $1628 \mathrm{~cm}^{-1}$, which are similar to peak patterns associated with $\beta$-sheet structure. ${ }^{20}$ This result can be compared to other depsipeptide systems, in which $\beta$-sheet folding is observed in the peptide counterpart but absent in the modified structure, ${ }^{29,25}$ suggesting that $\beta$ sheet interactions may be partially disrupted by the inclusion of ester bonds into the peptide backbone. Our results suggest that a higher order structure other than the more commonly reported $\beta$-sheet formation between peptide chains 
in Fmoc-peptide systems contributes to the self-assembly and gelation of Fmoc-D-Lac-K-A-8.

While the morphology of Fmoc-D-Lac-K-A-8 nanostructures, as shown by TEM, indicates the formation of micelles and fibers in water after 18 hours and 4 days respectively, the IR data on pre- and post-gelation do not provide any additional information to allow us to distinguish between different stages of self-assembly. Our data indicates, however, that the rate of selfassembly and subsequent gelation is decreased for hydrogen bonding-deficient materials such as the depsipeptides described herein.

\section{Helical stacking of the fluorenyl groups}

CD spectra were collected for Fmoc-D-Lac-K-A-8 after 4 days at $10 \mathrm{mg} \mathrm{ml} \mathrm{m}^{-1}$ in PBS $+\mathrm{NaCl}$, PBS, and water. At the time of the data collection, gelation was only observed in PBS $+\mathrm{NaCl}$. The spectra show positive dichroism from 260-302 $\mathrm{nm}$ for samples prepared in the salt containing solutions (Fig. 6A), evidence that self-assembly has proceeded to a greater extent in these solutions. CD data for Fmoc-DAKA-8 was collected after 24 hours in PBS $+\mathrm{NaCl}$, PBS, and water (Fig. 6), consistent with the time at which hydrogel formation is observed. The peptide was less soluble in the salt containing solutions and did not selfassemble to an extent that would form gels in PBS or PBS $+\mathrm{NaCl}$ at 5 or $2.5 \mathrm{mg} \mathrm{ml}^{-1}$. In water, gelation of Fmoc-DAKA-8 was observed after 18 hours, and peaks between $260 \mathrm{~nm}$ and $310 \mathrm{~nm}$ were observed (Fig. 6B). When observed pre-gelation, CD of Fmoc-DAKA-8 shows similar peak patterns observed in the gelled form of the peptide (data not shown), confirming that self-assembly has occurred prior to the point of gelation. A similar spectral feature, the positive peaks from $260-310 \mathrm{~nm}$, is observed for other Fmoc-peptide gel systems in which the fluorenyl groups are thought to be stacked in a helical manner. ${ }^{22}$
For both the depsipeptide and peptide, helical stacking of the fluorenyl group was observed in gelled forms or in samples very close to gelation. These results suggest that the helical stacking of fluorenyl groups is a critical step in the self-assembly process that precedes the onset of gelation and appears to be a process largely unaffected by ester substitutions in the peptide backbone.

\section{Hydrolytic degradation of Fmoc-D-Lac-K-A-8}

The stability of non-gelled and gelled samples of Fmoc-D-Lac-KA-8 was analyzed in PBS + $\mathrm{NaCl}$ and water (Fig. 7) using HPLCmass spectrometry (LC-MS). The degradation profiles in PBS (Fig. $\mathrm{S} 3 \dagger$ ) and $\mathrm{PBS}+\mathrm{NaCl}$ are comparable, thus we chose to compare stability between one ionic and aqueous solution for simplicity. Low concentration samples $\left(1 \mathrm{mg} \mathrm{ml}^{-1}\right)$ were used as the non-gelled components for both solutions, as gelation has only been observed at $10 \mathrm{mg} \mathrm{ml}^{-1}$. In PBS $+\mathrm{NaCl}$, the non-gelled sample is completely degraded after 10 days. In the gelled form, intact depsipeptide remains in the mixture after 14 days. The degradation products are most likely a result of hydrolysis of Fmoc-D-Lac-K-A-8 at the ester bonds, as the mass-to-charge $(\mathrm{m} / \mathrm{z})$ values associated with the new peaks in the chromatogram correlate to the predicted hydrolyzed structures (Fig. S4†). In water, the depsipeptide is relatively stable in both the non-gelled and gelled state, though it appears slightly less degraded in the gelled state. Within gelled samples, individual molecules are organized into supramolecular assemblies and therefore the depsipeptide backbone may be less accessible to water molecules and hydronium and hydroxide ions than in the solution state. Tian et al. observed nearly identical behaviour in solutions and gels from depsipeptides containing only one backbone ester substitution. ${ }^{25}$ It is unclear why degradation occurred faster in salt-containing solutions than in pure water;
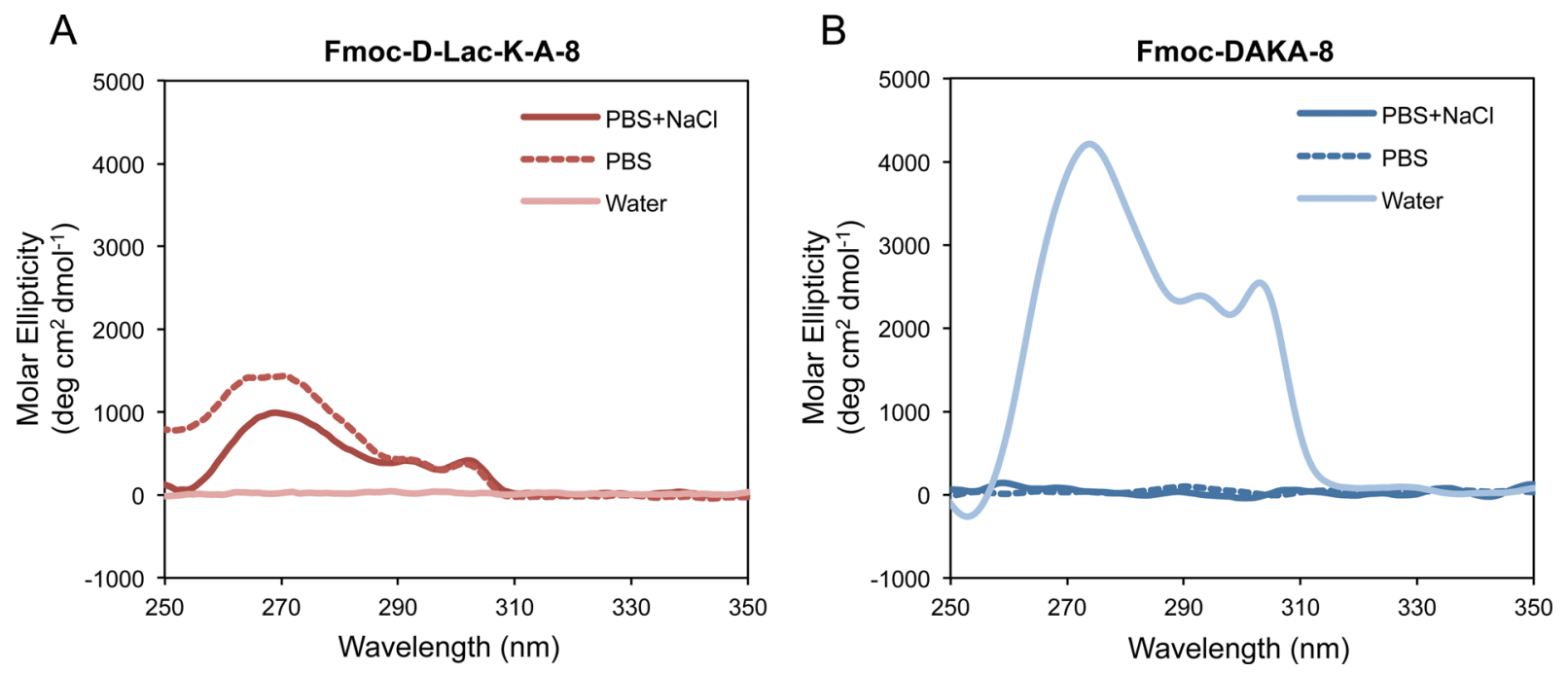

Fig. 6 CD spectra of Fmoc-D-Lac-K-A-8 in PBS + NaCl, PBS, and water (A) and Fmoc-DAKA-8, the peptide equivalent (B). The depsipeptide was prepared at $10 \mathrm{mg} \mathrm{ml}^{-1}$ and aged for 4 days. In PBS $+\mathrm{NaCl}$ and PBS, gels formed after 2 days and 6 days respectively. Strong absorbance peaks from 270-300 nm are observed, which have been reported to be helical stacking of the Fmoc-group. No gels formed in water at the time the CD was collected. Fmoc-DAKA-8 only exhibited gelation in water due to poor solubility in PBS and PBS $+\mathrm{NaCl}$, therefore only the CD spectrum of the peptide in water shows a similar positive dichroism from $270-300 \mathrm{~nm}$ indicating helical-like association of Fmoc groups. 

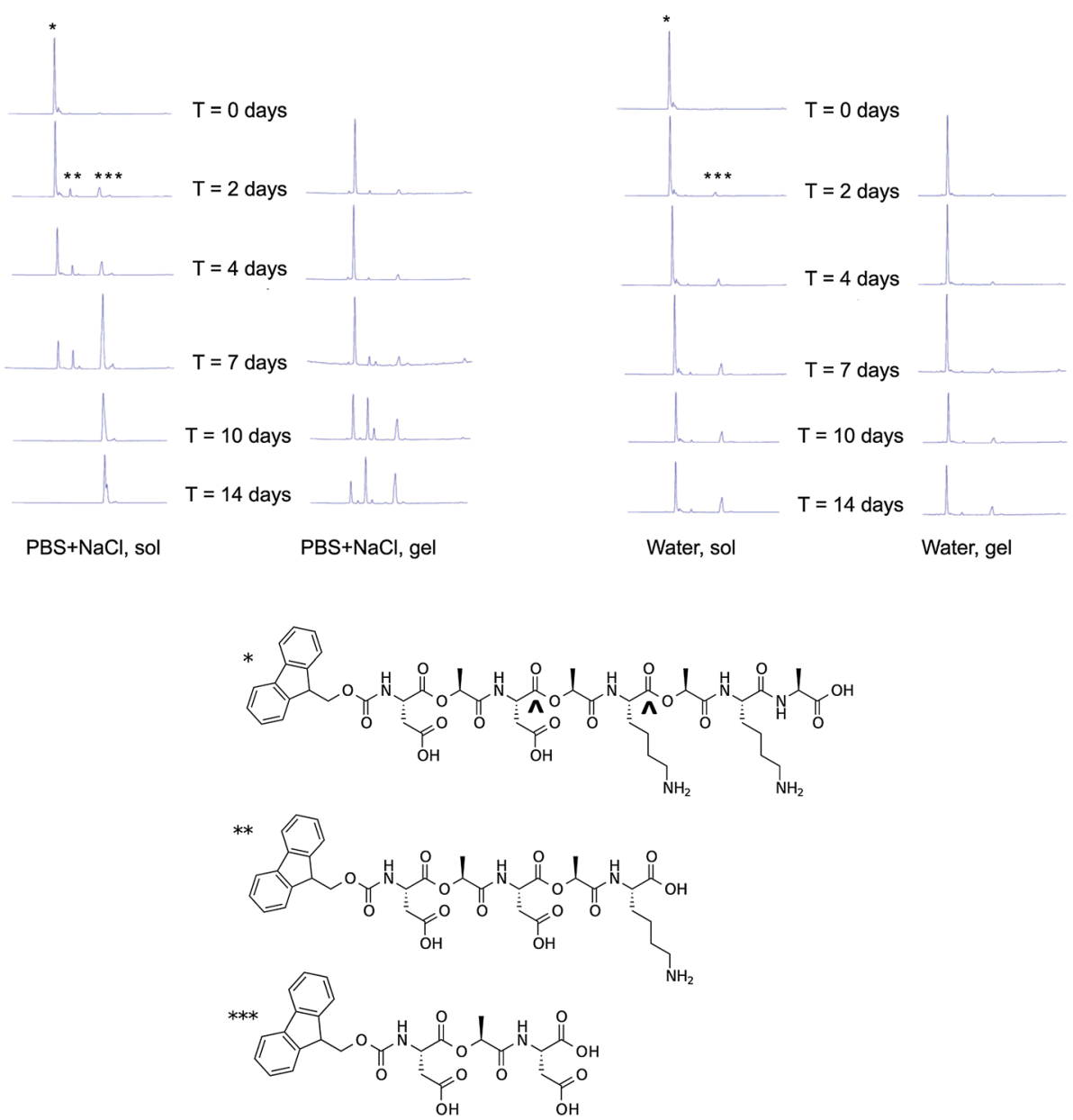

Fig. 7 Degradation of Fmoc-D-Lac-K-A-8 over time. Degradation of the depsipeptide was evaluated with HPLC-MS in PBS + NaCl (top left), and water (top right) in a non-gelled and a gelled sample. The chemical structure of Fmoc-D-Lac-K-A-8, represented by the single asterisk (*) is hydrolyzed at two potential ester sites $(\wedge)$, to yield the two predicted degradation products, represented by a double $(* *)$ and triple $(* * *)$ asterisk. As shown by peak intensity changes in the chromatograms over time, the depsipeptide in solution degrades after 2 days and is completely hydrolyzed in the presence of salt after 7 days. In the gelled form, the rate of hydrolysis is slower and is mostly degraded in PBS + NaCl after 14 days. In water, both the depsipeptide in solution and as a gel showed the slowest rate of gelation. The study conducted in PBS and the masses of these structures are provided in the ESI (Fig. S2 and S3†).

perhaps the charged ion species shield oppositely charged peptide side chains that would otherwise repulse hydronium and/or hydroxide ions responsible for ester hydrolysis.

\section{Effect of increasing electrostatic interactions or hydrophobicity on gelation}

Of all the structures investigated within our depsipeptide library, Fmoc-D-Lac-K-A-8 alone forms a hydrogel after 2 days, 6 days, or 3 weeks in PBS $+\mathrm{NaCl}$, PBS, and water, respectively. Fmoc-D-Lac-K-A-16 (Fig. S5A $\dagger$ ) was synthesized in an attempt to investigate the effect of increasing electrostatic interactions on self-assembly. No gelation was observed at $10 \mathrm{mg} \mathrm{ml}^{-1}$ in water, $\mathrm{PBS}$, or PBS $+\mathrm{NaCl}$, and the emission spectra show no unique patterns after 5 days (Fig. S5B $\dagger$ ). In another attempt, we increased the overall hydrophobicity of the structure by adding a phenylalanine (F) to synthesize Fmoc-D-Lac-K-F-8. Gelation was observed in water within minutes at 5,10 , and $20 \mathrm{mg} \mathrm{ml}^{-1}$. As a comparison, the mechanical properties of Fmoc-D-Lac-K-A-
8 and Fmoc-D-Lac-K-F-8 gels were analyzed using parallel-plate rheometry (Table 2). Results show that increasing the overall hydrophobicity of the depsipeptide increases the gel strength, a trend that has been reported in self-assembling Fmoc-dipeptide systems. ${ }^{16}$

Fmoc-D-Lac-K-F-8 was less soluble in PBS and PBS $+\mathrm{NaCl}$ than in water and did not form a hydrogel in the salt-containing solutions even after 7 days. The observed fluorescence emission peaks at $310 \mathrm{~nm}$ in $\mathrm{PBS}$ and $\mathrm{PBS}+\mathrm{NaCl}$ (Fig. 8) reflect the

Table 2 Gelation properties of depsipeptides. Increasing the overall hydrophobicity increases gel strength

\begin{tabular}{|c|c|c|c|c|}
\hline Depsipeptide & $\begin{array}{l}\text { Concentration } \\
\left(\mathrm{mg} \mathrm{ml}^{-1}\right)\end{array}$ & Solvent & $\mathrm{G}^{\prime}(\mathrm{Pa})$ & $\mathrm{G}^{\prime \prime}(\mathrm{Pa})$ \\
\hline $\begin{array}{l}\text { Fmoc-D- } \\
\text { Lac-K-A-8 }\end{array}$ & 10 & $\begin{array}{l}\mathrm{PBS}+ \\
\mathrm{NaCl}\end{array}$ & $34 \pm 19$ & $9 \pm 3$ \\
\hline Fmoc-D- & 5 & Water & $106 \pm 23$ & $12 \pm 2$ \\
\hline
\end{tabular}




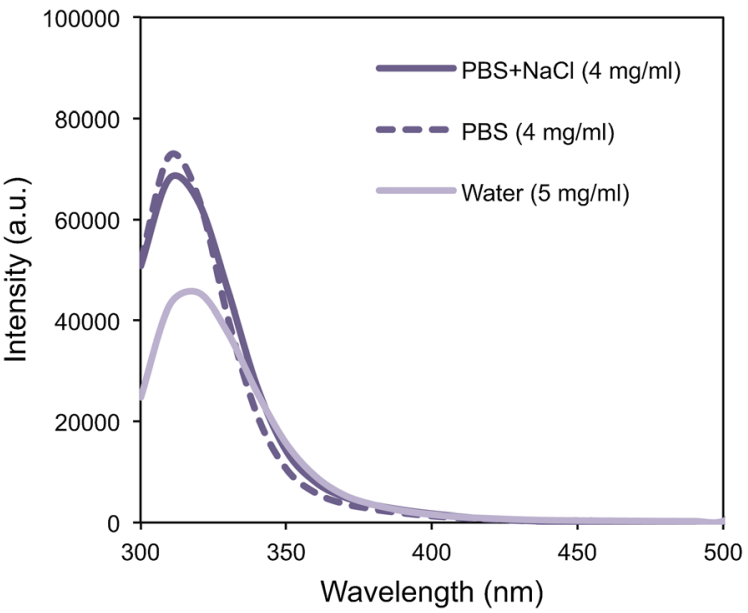

Fig. 8 Fluorescence emission spectra of Fmoc-D-Lac-K-F-8. In water, a peak at $320 \mathrm{~nm}$ is observed and the depsipeptide also forms a gel under those conditions. The observed peaks at $310 \mathrm{~nm}$ in PBS and $\mathrm{PBS}+\mathrm{NaCl}$ reflect the absorbance of the fluorenyl group in solution, as gels did not form under these conditions.

absorbance of the fluorenyl group in solution, and does not correlate to a state of ordered assembly. In water, the association of Fmoc-groups is close but less extensively stacked as observed with the less hydrophobic Fmoc-D-Lac-K-A-8. J-aggregate features have been observed in the emission spectrum of Fmoc-Phe-Phe-OH gels, suggesting that the phenylalanine residue interacts with the fluorenyl group. ${ }^{21}$ This absorbance was not detected in our system, suggesting that when FmocD-Lac-K-F-8 self-assembles into a fiber-like nanostructure, the phenylalanine ring does not associate closely with the Fmocgroup. The chemical backbone of the depsipeptide differs from Fmoc-Phe-Phe-OH in length, incorporation of esters, and the fact that it is self-complementary. All of these effects have been reported to affect self-assembly in other Fmoc-peptide systems, ${ }^{29,19}$ thus, we would not expect our results to directly trend with a system limited by two peptide residues.

\section{Importance of fluorenyl association for Fmoc-depsipeptide gelation}

The self-assembled structures of well-studied peptide families have been reported in the literature and rely heavily on spectroscopic data that correlate to specific secondary folding characteristics in better-characterized protein systems. Observations of secondary structures were absent in the analysis of Fmoc-D-Lac-K-A-8 because such interpretation with CD and IR are based on the chemical features of the native peptide backbone. The incorporation of esters within the depsipeptides disrupts both the flexibility of the structures and their ability to hydrogen bond, thus the comparison of depsipeptides to peptides may be limited with these techniques. Our results suggest that depsipeptides assemble into ordered structures, such as fibers and hydrogels, and the close association of fluorenyl groups is an important indicator of self-assembly and gelation of our materials.
The gelation of our family of Fmoc-depsipeptides is influenced by the sequence of charged residues along the chemical backbone, the presence of salt, and the overall hydrophobicity of the structure. Fmoc-D-Lac-K-A-8 formed fibers and hydrogels in water, PBS, and PBS + NaCl. The structural data reveals that the fluorenyl groups are aligned in a closely associated manner in gelled samples, and the data is consistent with Fmoc-peptides that show helical stacking of the fluorenyl groups. Replacing alanine residues with lactic acid contributes to a loss of hydrogen bonding ability due to ester inclusion, which may contribute to slowing the self-assembly process and therefore extend the time to the onset of gelation. The peptide Fmoc-DAKA-8 forms a gel in water overnight, while the depsipeptide Fmoc-D-Lac-K-A-8 in the same solution forms a gel in 3 weeks. Salt-containing solutions most likely act to shield the charged residues, which may help to drive the self-assembly of Fmoc-D-Lac-K-A-8 more rapidly in PBS + $\mathrm{NaCl}$ than in PBS or water. Incorporating a phenylalanine residue to the depsipeptide, Fmoc-D-Lac-K-F-8, leads to hydrogel formation in water within minutes, suggesting that hydrophobic interactions may facilitate rapid self-assembly leading to gel formation in the absence of side-chain shielding salts. The hydrogen bonding sites are reduced due to the presence of the ester bond, however this structural modification does not completely disrupt self-assembly and subsequent gelation.

\section{Conclusions}

In this work, we synthesized and assayed the self-assembly and gelation capability of a small family of Fmoc-protected depsipeptide oligomers. It is clear that incorporation of regular esters into a native peptide backbone does not prohibit the selfassembly into supramolecular nanostructures that in some cases, form networks dense and entangled enough to cause gelation in water. We have demonstrated that the self-assembly of Fmocdepsipeptides leading to hydrogel formation is dependent on charge, sequence, and salt concentration. The close association and possibly helical stacking of fluorenyl groups, as determined by fluorescence emission and CD spectroscopies, corresponds with the onset of self-assembly and precedes gelation, and the rate at which the self-assembled fibrillar networks become dense enough to form gels is dependent on the ionic character of the solution. Depsipeptide molecules within gel-forming supramolecular assemblies are protected from degradation relative to those in solution, and the presence of salt increases the hydrolysis rate of the depsipeptide molecules at the ester bond sites. Within our system, we have found that increasing the overall hydrophobicity of the structure increases both gelation rate and gel strength. Our depsipeptide library can therefore be tailored to control the formation rate and strength of the self-assembled gels, allowing them to be applied to a broad range of biomedical applications requiring materials with hydrolytic susceptibility, such as tissue scaffolds and drug delivery systems.

\section{Acknowledgements}

The authors would like to thank Dr Lauren Webb, Annette Raigoza, Christina Ragain, and Jason Dugger for access and use 
of their FTIR; Dr Brent Iverson, Dr John Hardy, and Cameron Peebles for insightful discussions; and Ryan Stowers for his thoughtful review of this manuscript. This work was supported by the Welch Foundation and the National Institutes of Health (R21HL102806).

\section{References}

1 M. E. Davis, J. P. M. Motion, D. A. Narmoneva, T. Takahashi, D. Hakuno, R. D. Kamm, S. G. Zhang and R. T. Lee, Circulation, 2005, 111, 442-450.

2 A. L. Sieminski, A. S. Was, G. Kim, H. Gong and R. D. Kamm, Cell Biochem. Biophys., 2007, 49, 73-83.

3 T. C. Holmes, S. de Lacalle, X. Su, G. S. Liu, A. Rich and S. G. Zhang, Proc. Natl. Acad. Sci. U. S. A., 2000, 97, 67286733.

4 Z. L. Luo, B. Akerman, S. G. Zhang and B. Norden, Soft Matter, 2010, 6, 2260-2270.

5 Y. Loo, S. Zhang and C. A. E. Hauser, Biotechnol. Adv., 2012, 30, 593-603.

6 Y. Yang, U. Khoe, X. Wang, H. Akihiro, Y. Hidenori and S. Zhang, Nano Today, 2009, 4, 193-210.

7 Z. L. Luo, X. J. Zhao and S. G. Zhang, Macromol. Biosci., 2008, 8, 785-791.

8 S. G. Zhang, T. C. Holmes, C. M. Dipersio, R. O. Hynes, X. Su and A. Rich, Biomaterials, 1995, 16, 1385-1393.

9 A. Saiani, A. Mohammed, H. Frielinghaus, R. Collins, N. Hodson, C. M. Kielty, M. J. Sherratt and A. F. Miller, Soft Matter, 2009, 5, 193-202.

10 M. R. Caplan, E. M. Schwartzfarb, S. G. Zhang, R. D. Kamm and D. A. Lauffenburger, Biomaterials, 2002, 23, 219-227.

11 Y. Loo, S. G. Zhang and C. A. E. Hauser, Biotechnol. Adv., 2012, 30, 593-603.

12 F. Gelain, A. Horii and S. G. Zhang, Macromol. Biosci., 2007, 7, 544-551.

13 A. Horii, X. Wang, F. Gelain and S. Zhang, PLoS One, 2007, 2, e190.

14 C. A. E. Hauser and S. Zhang, Chem. Soc. Rev., 2010, 39, 27802790.

15 D. J. Adams, Macromol. Biosci., 2011, 11, 160-173.

16 D. J. Adams, L. M. Mullen, M. Berta, L. Chen and W. J. Frith, Soft Matter, 2010, 6, 1971-1980.

17 D. J. Adams and P. D. Topham, Soft Matter, 2010, 6, 3707-3721.

18 H. Wang, Z. Yang and D. J. Adams, Mater. Today, 2012, 15, 500-507.

19 X. D. Xu, C. S. Chen, B. Lu, S. X. Cheng, X. Z. Zhang and R. X. Zhuo, J. Phys. Chem. B, 2010, 114, 2365-2372.

20 A. M. Smith, R. J. Williams, C. Tang, P. Coppo, R. F. Collins, M. L. Turner, A. Saiani and R. V. Ulijn, Adv. Mater., 2008, 20, 37-41.
21 C. Tang, R. V. Ulijn and A. Saiani, Langmuir, 2011, 27, 1443814449.

22 V. Jayawarna, M. Ali, T. A. Jowitt, A. E. Miller, A. Saiani, J. E. Gough and R. V. Ulijn, Adv. Mater., 2006, 18, 611-614.

23 X. Mu, K. M. Eckes, M. M. Nguyen, L. J. Suggs and P. Ren, Biomacromolecules, 2011, 13, 3562-3571.

24 S. Rotem and A. Mor, Biochim. Biophys. Acta, Biomembr., 2009, 1788, 1582-1592.

25 Y. F. Tian, G. A. Hudalla, H. Han and J. H. Collier, Biomater. Sci., 2013, 1, 1037-1045.

26 T. Ohyama, H. Oku, M. Yoshida and R. Katakai, Biopolymers, 2001, 58, 636-642.

27 H. Oku, T. Ohyama, A. Hiroki, K. Yamada, K. Fukuyama, H. Kawaguchi and R. Katakai, Biopolymers, 2004, 75, 242254.

28 X. Y. Yang, M. Wang and M. C. Fitzgerald, Bioorg. Chem., 2004, 32, 438-449.

29 R. C. Elgersma, T. Meijneke, G. Posthuma, D. T. S. Rijkers and R. M. J. Liskamp, Chem.-Eur. J., 2006, 12, 3714-3725.

30 M. Altman, P. Lee, A. Rich and S. G. Zhang, Protein Sci., 2000, 9, 1095-1105.

31 F. Gelain, A. Lomander, A. L. Vescovi and S. G. Zhang, J. Nanosci. Nanotechnol., 2007, 7, 424-434.

32 S. G. Zhang, T. Holmes, C. Lockshin and A. Rich, Proc. Natl. Acad. Sci. U. S. A., 1993, 90, 3334-3338.

33 S. G. Zhang, Biotechnol. Adv., 2002, 20, 321-339.

34 S. Zhang, D. M. Marini, W. Hwang and S. Santoso, Curr. Opin. Chem. Biol., 2002, 6, 865-871.

35 X. J. Zhao and S. G. Zhang, Chem. Soc. Rev., 2006, 35, 11051110.

36 D. M. Ryan, S. B. Anderson, F. T. Senguen, R. E. Youngman and B. L. Nilsson, Soft Matter, 2011, 6, 475-479.

37 J. F. Smith, T. P. J. Knowles, C. M. Dobson, C. E. MacPhee and M. E. Welland, Proc. Natl. Acad. Sci. U. S. A., 2006, 103, 15806-15811.

38 M. M. Nguyen, N. Ong and L. Suggs, Org. Biomol. Chem., 2013, 11, 1167-1170.

39 J. W. Sadownik, J. Leckie and R. V. Ulijn, Chem. Commun., 2011, 47, 728-730.

40 I. F. Gallardo and L. J. Webb, Langmuir, 2012, 28, 3510-3515. 41 D. Schweitzer, K. H. Hausser and M. W. Haenel, Chem. Phys., 1978, 29, 181-185.

42 Z. Yang, H. Gu, Y. Zhang, L. Wang and B. Xu, Chem. Commun., 2004, 208-209.

43 H. K. Kang, D. E. Kang, B. H. Boo, S. J. Yoo, J. K. Lee and E. C. Lim, J. Phys. Chem. A, 2005, 109, 6799-6804.

44 S. Fleming, S. Debnath, P. W. J. M. Frederix, T. Tuttle and R. V. Ulijn, Chem. Commun., 2013, 49, 10587-10589.

45 H. M. Wang, C. H. Yang, M. Tan, L. Wang, D. L. Kong and Z. M. Yang, Soft Matter, 2011, 7, 3897-3905. 